\title{
Evaluation of Crushed Ticagrelor Tablet Doses: Recovery Following Crushing and Naso-gastric Tube Passage Ex Vivo
}

\author{
Barry Crean - Cindy Finnie - Anna Crosby
}

Published online: 5 June 2013

(c) The Author(s) 2013. This article is published with open access at Springerlink.com

\begin{abstract}
Background Orally available ticagrelor in combination with low-dose aspirin $(75-100 \mathrm{mg} /$ day $)$ is indicated for adult patients with acute coronary syndromes. However, patients with swallowing difficulties may be unable to consume the currently available $90-\mathrm{mg}$ tablet. It is hypothesized that ticagrelor could be given to this patient cohort as a crushed dose administered either orally or via a naso-gastric (NG) tube.

Objectives To investigate the potential use of crushed ticagrelor tablets (90- and 180-mg doses) for oral dose or NG tube administration.

Methods Ticagrelor tablets (90 or $180 \mathrm{mg}$ [two 90-mg tablets]) were prepared to emulate oral and NG tube administration by similar methods. For the oral dose, ticagrelor tablets were crushed using a mortar and pestle and transferred to a dosing cup. $100 \mathrm{~mL}$ of water was added to the mortar, stirred, and the contents were transferred to the dosing cup and stirred to form a suspension. At this stage, where the suspension would normally be administered to a patient, it was collected for high performance liquid chromatography (HPLC) analysis. The mortar was then flushed with $100 \mathrm{~mL}$ of water, and the contents were again
\end{abstract}

A. Crosby was formerly at AstraZeneca, Charter Way, Macclesfield, UK.

B. Crean $(\bowtie) \cdot$ C. Finnie

AstraZeneca, Research \& Development, Pharmaceutical

Development, Silk Road Business Park, Charter Way,

Hurdsfield Industrial Estate, Macclesfield SK10 2NA, UK

e-mail: barry.crean@astrazeneca.com

A. Crosby

Department of Chemistry, School Life Science,

University of Sussex, East Sussex, UK transferred to the dosing cup, stirred, and collected for HPLC analysis. For the NG dose, polyvinylchloride, polyurethane, and silicone size CH10 NG tubes were used. The tablets were crushed using a mortar and pestle, diluted with $50 \mathrm{~mL}$ of water, and stirred. At this stage, where the suspension would normally be administered to a patient through an NG tube using a syringe, it was collected for HPLC analysis. The mortar was then flushed with two additional $50 \mathrm{~mL}$ aliquots of water and the contents were passed through the NG tube. HPLC analysis examined the recoverability of ticagrelor in each of the dose suspensions and flushes and the stability of the suspension when held in a syringe for up to $2 \mathrm{~h}$.

Results One or two crushed 90-mg ticagrelor tablets, prepared for either oral or NG tube administration, delivers a mean dose of $\geq 97 \%$ of the original tablet. No degradation of the suspensions was detected after ticagrelor had been held in the syringe for up to $2 \mathrm{~h}$.

Conclusion Although not an approved method of administration, these results suggest that ticagrelor tablets can be crushed and prepared for oral administration or for administration via an NG tube. From a clinical perspective, a syringe hold-time of up to $2 \mathrm{~h}$ should allow for enough time between preparation and administration (orally or via an NG tube) of the dispersed tablets to the patient. Future studies are required to test the effect of crushed dosing on pharmacokinetic and pharmacodynamic parameters.

\section{Introduction}

Acute coronary syndromes (ACS) encompass a range of myocardial ischemic events that represent a significant clinical concern worldwide [1, 2]. ACS is typically categorized as either ST segment elevation (STE-) ACS or 
non-STE ACS (NSTE-ACS), and NSTE-ACS can be further categorized into non-STE myocardial infarction and unstable angina [1]. The current recommended treatment for patients with ACS is dual antiplatelet therapy; aspirin is taken in combination with another agent to inhibit platelet activity, preventing the development of this condition [3].

The thienopyridines, clopidogrel and prasugrel, are oral antiplatelet drugs that irreversibly inhibit the $\mathrm{P}_{2} \mathrm{Y}_{12}$ purinoreceptor [4], whereas ticagrelor, a first-in-class cyclopentyltriazolopyrimidine, is a reversibly binding, oral $\mathrm{P} \mathrm{Y}_{12}$ receptor antagonist [5]. Pharmacologic studies have shown that ticagrelor has a rapid onset of activity and enhanced inhibition of platelet aggregation compared with clopidogrel [6-8]. In addition, the large phase III PLATelet inhibition and patient Outcomes (PLATO) clinical trial has also reported that ticagrelor compared with clopidogrel significantly reduces the incidence of myocardial infarction, stroke, or death from vascular causes without an increase in the rates of major bleeding in patients with ACS [9].

Ticagrelor (180-mg loading dose, $90 \mathrm{mg}$ twice daily) is currently recommended for combination antiplatelet treatment with low-dose aspirin (150-300-mg loading dose, $75-100 \mathrm{mg}$ a day) for patients with ACS [1, 3, 10]. Most $\mathrm{P}^{2} \mathrm{Y}_{12}$ inhibitors used in ACS treatment, including ticagrelor, are only available in an oral form. This limitation represents a potential concern for patients with difficulty swallowing tablets, which in the general population may be as high as $40 \%$ of all adults $[11,12]$. In the elderly, swallowing difficulties are even more prevalent; nearly $60 \%$ of individuals (ages 60-89 years) indicate they have difficulties in swallowing tablets/capsules [13]. Difficulties with swallowing can also lead to noncompliance with treatment medication. Of those adults in the general population with swallowing difficulties, $14 \%$ reported that they have delayed taking their prescribed medication and $8 \%$ reported that they have skipped their medication entirely [11, 12]. In the elderly population, $68 \%$ of individuals with swallowing difficulties reported they had to crush or open a tablet in order to swallow the medication and $69 \%$ reported they have missed dose(s) because the tablet/capsule was too difficult to swallow [13]. In addition to patients with swallowing difficulties, patients who are unconscious when they arrive in the emergency room or during their hospital stay cannot take oral medications. For these individuals, an alternative method of administration is also necessary.

Studies have demonstrated that certain tablets can be administered through naso-gastric (NG) and gastrostomy tubes using a syringe [14]. In fact, one study demonstrated that crushed tablets of clopidogrel can be mixed with water and flushed down an NG feeding tube [14]. Despite the potential effects on the pharmacokinetics of the drug, it has been suggested that this route of delivery will be unlikely to cause any adverse events and may therefore provide a viable alternative to oral tablets for patients with swallowing difficulties [14].

The present ex vivo study was designed to investigate the potential use of ticagrelor as a crushed dose and prepared to emulate administration orally or via an NG tube.

\section{Materials and Methods}

Standard 90-mg ticagrelor tablets were prepared by similar methods to emulate oral and NG tube administration; two doses (90 and $180 \mathrm{mg}$ [two 90-mg tablets]) of ticagrelor were examined. For each method, one or two tablets were placed into a heavy glass mortar and crushed for $60 \mathrm{~s}$ with a glass pestle to form a powder. Purified water was used to disperse the crushed tablets.

\subsection{Oral Dose Administration}

A schematic diagram of oral dose administration is shown in Fig. 1. A ticagrelor tablet was placed in a mortar and crushed for $60 \mathrm{~s}$ using a pestle. The crushed tablet was transferred to a dosing cup, ensuring that all powder was transferred and none remained on the mortar and pestle. $100 \mathrm{~mL}$ of purified water was added to the mortar and stirred for $60 \mathrm{~s}$ using the pestle. The total contents of the mortar were transferred to the dosing cup and stirred for an additional $60 \mathrm{~s}$ using the pestle to ensure that all powder was dispersed. The mortar was flushed with another $100 \mathrm{~mL}$ of purified water and stirred for $30 \mathrm{~s}$ using the pestle. The total contents were transferred to another dosing cup and stirred for another $30 \mathrm{~s}$ to ensure that all remaining tablet particles were dispersed. Each of the suspensions, which would normally be administered to a patient from the dosing cup, was collected for high performance liquid chromatography (HPLC) analysis of drug recoverability.

\subsection{NG Dose Administration}

A schematic diagram of NG dose administration is shown in Fig. 2. Three types of NG tube were used in the study: polyvinylchloride (PVC), polyurethane (PUR), and silicone. PUR and PVC tubes were $110 \mathrm{~cm}$ in length, silicone tubes were $85 \mathrm{~cm}$ in length and all tubes were size CH10. Each NG tube was flushed with $25 \mathrm{~mL}$ of purified water using a $50-\mathrm{mL}$ PVC oral enteral syringe. Ticagrelor tablets ( 90 or $180 \mathrm{mg}$ [two 90-mg tablets]) were placed in a mortar and crushed for $60 \mathrm{~s}$ using a pestle. $50 \mathrm{~mL}$ of purified water (for both the 90 and 180-mg doses) was added to the mortar and stirred for $60 \mathrm{~s}$ using the pestle. The suspension was taken from the 
Fig. 1 Schematic diagram of oral administration. $H P L C$ high performance liquid chromatography

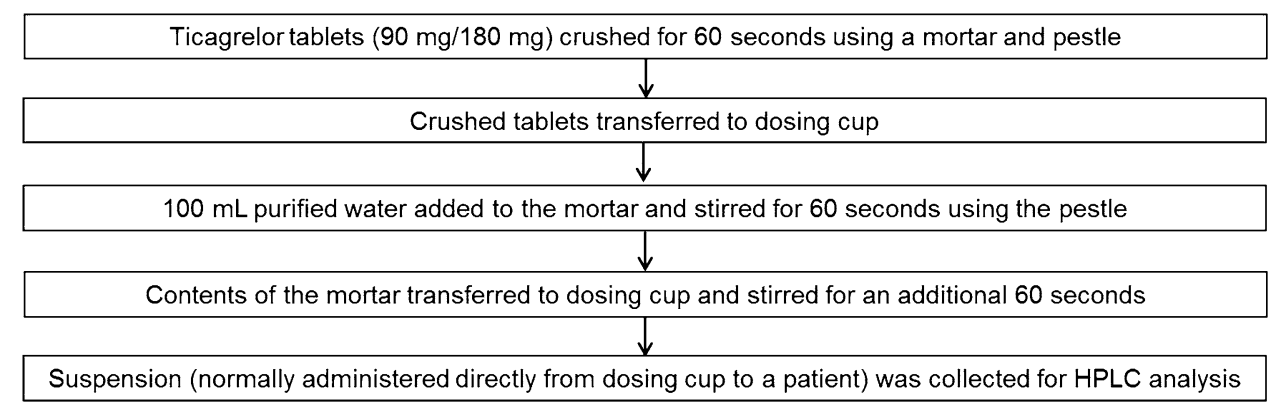

Suspension (normally administered directly from dosing cup to a patient) was collected for HPLC analysis

Then $100 \mathrm{~mL}$ purified water added to the mortar and stirred for 30 seconds using the pestle

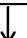

Contents of the mortar transferred to dosing cup and stirred for an additional 30 seconds

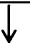

Suspension (normally administered directly from dosing cup to a patient) was collected for HPLC analysis

NG tubes flushed with $25 \mathrm{~mL}$ purified water using a $50 \mathrm{~mL}$ PVC oral enteral syringe

\begin{tabular}{|l|}
\hline Ticagrelor tablets $(90 \mathrm{mg} / 180 \mathrm{mg}$ ) crushed for 60 seconds using a mortar and pestle \\
\hline $50 \mathrm{~mL}$ purified water added to the mortar and stirred for 60 seconds using the pestle \\
\hline \\
Suspension taken from the mortar with a $50 \mathrm{~mL}$ PVC oral enteral syringe \\
\hline \\
Syringe was connected to the NG tube at the Luer-lock connection \\
and the suspension passed through the NG tube and collected for HPLC analysis
\end{tabular}

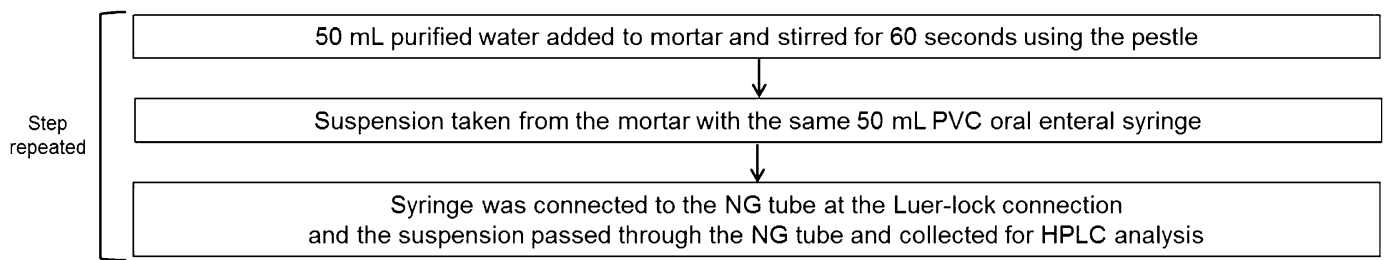

$25 \mathrm{~mL}$ purified water flushed through the NG tube using the same $50 \mathrm{~mL}$ PVC oral enteral syringe

Fig. 2 Schematic diagram of NG tube administration. $N G$ naso-gastric, $H P L C$ high performance liquid chromatography, $P V C$ polyvinylchloride

mortar using a 50-mL PVC oral enteral syringe, which was then connected to the NG tube at the Luer-lock connection, and the contents, which would normally be administered to a patient at this stage, were passed through the NG tube and collected for HPLC analysis of drug recoverability. Another $50 \mathrm{~mL}$ of purified water was added to the mortar and the contents were stirred with the pestle for $60 \mathrm{~s}$. The suspension was removed from the mortar using the same $50-\mathrm{mL}$ oral enteral syringe, which was again connected to the NG tube at the Luer-lock connection, and the contents were passed through the NG tube and collected for HPLC analysis. Another $50 \mathrm{~mL}$ of purified water was added to the mortar and stirred with the pestle for $60 \mathrm{~s}$. The suspension was again removed from the mortar using the same $50-\mathrm{mL}$ oral enteral syringe, which was connected to the NG tube at the Luerlock connection, and the contents were passed through the
NG tube and collected for HPLC analysis. Finally, using the same 50-mL PVC oral enteral syringe, $25 \mathrm{~mL}$ of purified water was flushed through the NG tube. Each of the collected flushes was analyzed separately by HPLC.

\subsection{Sample Analysis}

In order to determine the recoverability of ticagrelor, the flushes for each method were prepared for HPLC analysis and collected in volumetric flasks. The collected oral doses and flushes were each diluted up to $200 \mathrm{~mL}$ with 70/30 (volume/volume [v/v]) acetonitrile/water; the collected NG tube doses and flushes were each diluted up to $100 \mathrm{~mL}$ with 70/30 (v/v) acetonitrile/water. A 10-mL aliquot of the 180-mg samples was diluted up to $20 \mathrm{~mL}$ with $35 / 65$ (v/v) acetonitrile/water. The concentration of ticagrelor was 
determined by comparing the values from HPLC analysis of the ticagrelor sample with values from a ticagrelor reference standard solution prepared at a similar nominal concentration and analyzed in the same way. The ticagrelor sample and reference standard solutions were analyzed by isocratic reversed phase HPLC and ultraviolet detection using appropriate column and chromatographic conditions. The amount of drug recovered was expressed as a percentage of the total intact ticagrelor dose (either 90 or $180 \mathrm{mg}$ [label claim]). Experiments for each method were repeated three times. The results were expressed as the mean percentage of recovery of the intact dose. Release testing, including measurement of ticagrelor tablet content uniformity, was performed using standardized methods.

The in-use stability of the aqueous suspensions of ticagrelor tablets (90- and 180-mg doses) held within the 50-mL PVC oral enteral syringe for up to $2 \mathrm{~h}$ (i.e., 0,1 , and $2 \mathrm{~h})$ was examined. HPLC analysis measured the degradation products.

\section{Study Endpoints}

The primary endpoint of the study was the mean percentage of ticagrelor recovered from the samples compared with the intact tablet dose for each method of administration, for both the 90- and 180-mg ticagrelor doses. Recovery was considered acceptable if the average recovery exceeded $95 \%$.

The in-use stability of aqueous suspensions of ticagrelor tablets, in terms of the observed level of degradation, was also quantified.

\section{Results}

Data for the mean percentage recovery of ticagrelor are shown in Table 1.

Table 1 Mean percentage recovery of ticagrelor following oral and NG tube administration

\begin{tabular}{|c|c|c|}
\hline $\begin{array}{l}\text { Administration } \\
\text { method }\end{array}$ & $\begin{array}{l}90-\mathrm{mg} \text { dose } \\
\text { Mean \% recovery } \\
\text { [range] }^{\mathrm{a}}\end{array}$ & $\begin{array}{l}\text { 180-mg dose } \\
\text { Mean \% recovery } \\
\text { [range] }^{\text {a }}\end{array}$ \\
\hline Crushed oral dose & 99.47 [98.43-100.08] & $99.20[98.19-100.05]$ \\
\hline \multicolumn{3}{|l|}{ NG tube } \\
\hline PVC & 99.12 [97.86-100.68] & 97.25 [96.45-97.98] \\
\hline PUR & 100.43 [95.28-103.89] & 97.92 [97.26-98.97] \\
\hline Silicone & $101.37^{\mathrm{b}}[96.92-114.47]$ & 97.29 [97.06-97.42] \\
\hline
\end{tabular}

$N G$ naso-gastric, $P U R$ polyurethane, $P V C$ polyvinylchloride

a Average of three experiments

b Average of five experiments
An acceptable level of recovery was reported for the 90and 180-mg doses for both routes of administration. For the 90-mg dose, silicone NG tubes provided a mean recovery of $101 \%$ (mean range 97-115\%), whereas PUR NG tubes provided a mean recovery of $100 \%$ (mean range 95-104 \%) and PVC NG tubes provided a mean recovery of $99 \%$ (mean range 98-101\%). The results for the 180 -mg dose for all three types of NG tube were similar (mean range 97-98\%) as were results for the 90- and 180-mg crushed oral doses (mean range 98-100\%). Recovery across administration methods was higher for the 90-mg doses of ticagrelor, compared with the 180-mg doses.

There were no signs of degradation (i.e., any individual degradation product $<0.2 \%$ weight/weight [w/w] and total degradation products $<0.5 \% \mathrm{w} / \mathrm{w}$ ) in the 90 - and 180-mg suspensions of ticagrelor when retained in a syringe for up to $2 \mathrm{~h}$.

\section{Discussion}

The recommended treatment for ACS is dual antiplatelet therapy, and while it is effective [9, 15-17], it is often challenging to administer the indicated dose to patients who have difficulty swallowing. An alternative method of oral administration, which circumvents the need to swallow whole tablets, would provide an alternative option for these patients.

Results from the current study demonstrated that crushed tablets prepared to emulate oral or NG tube administration may provide patients with an acceptable method of delivery of their ticagrelor dose. Results were uniform for each route of delivery and for all three types of $\mathrm{NG}$ tubes, and demonstrated greater than $97 \%$ mean recoverability of the original dose. Release testing demonstrated that the 90-mg ticagrelor tablets exhibited acceptable content uniformity (acceptance value $=4.07$, individual tablet assay range 98.6-104.6\%). This variability in individual tablet content uniformity may have contributed to the relatively high individual dose recovery value reported (114.47\%, Table 1).

The NG tubes investigated in this study were selected to ensure compatibility with a range of tube materials used in current clinical practice. Due to its small internal diameter relative to other available tubes, the size of tube chosen for this study (CH10) was considered to be worst-case with respect to blockage or accumulation of material; therefore, tubes of equivalent or greater size can potentially be used for this method of administration.

Suspensions of ticagrelor held for up to $2 \mathrm{~h}$ in the syringe did not show signs of degradation in this study. This may be an important factor in clinical practice, as the 
amount of time required to prepare and administer a crushed dose of ticagrelor to a patient should fall well within this timespan.

\section{Conclusion}

Although the routes of administration reported in this study are not approved for ticagrelor, the results suggest that ticagrelor tablets can be crushed and prepared for oral and NG tube administration. Furthermore, previous studies conducted in healthy volunteers and using electronic sensory testing equipment have failed to indicate that ticagrelor has any adverse or unpalatable taste [18, 19]. Future studies are required to test the effect of crushed dosing on pharmacokinetic and pharmacodynamic parameters.

Acknowledgments Funding: This study was sponsored by AstraZeneca Macclesfield, UK. Editorial assistance was provided by Tara N Miller, PhD, Tom Gallagher, $\mathrm{PhD}$, and Josh Collis on behalf of Gardiner-Caldwell Communications in the preparation of this article, funded by AstraZeneca. The Open Access fee was paid for by AstraZeneca.

Conflicts of Interest: Barry Crean and Cindy Finnie are employees of AstraZeneca. Anna Crosby was a previous employee of AstraZeneca.

Author Contributions: Barry Crean and Cindy Finnie were involved in the study design and interpretation of the data, and Anna Cosby was involved in data collection. Barry Crean, Cindy Finnie, and Anna Crosby were involved in the preparation, review, and approval of the manuscript, and confirm that all data are accurately represented.

Open Access This article is distributed under the terms of the Creative Commons Attribution Noncommercial License which permits any noncommercial use, distribution, and reproduction in any medium, provided the original author(s) and the source are credited.

\section{References}

1. Hamm CW, Bassand J-P, Agewall S, et al. ESC guidelines for the management of acute coronary syndromes in patients presenting without persistent ST-segment elevation. Eur Heart J. 2011;32: 2999-3054.

2. Lloyd-Jones D, Adams RJ, Brown TM, et al. Heart disease and stroke statistics 2010 update: a report from the American Heart Association. Circulation. 2010;121:e46-215.

3. Writing Committee Members, Jneid H, Anderson JL, Wright RS, et al. 2012 ACCF/AHA focused update of the guideline for the management of patients with unstable angina/non-ST-elevation myocardial infarction (updating the 2007 guideline and replacing the 2011 focused update): a report of the American College of Cardiology Foundation/American Heart Association Task Force on practice guidelines. Circulation. 2012;126:875-910.
4. Storey RF. Biology and pharmacology of the platelet P2Y12 receptor. Curr Pharmaceut Design. 2006;12:1255-9.

5. Husted S. Evaluating the risk-benefit profile of the direct-acting P2Y12 inhibitor ticagrelor in acute coronary syndromes. Postgrad Med. 2011;123:79-90.

6. Gurbel PA, Bliden KP, Butler K, et al. Randomized double-blind assessment of the ONSET and OFFSET of the antiplatelet effects of ticagrelor versus clopidogrel in patients with stable coronary artery disease: the ONSET/OFFSET study. Circulation. 2009; 120:2577-85.

7. Husted S, Emanuelsson H, Heptinstall S, et al. Pharmacodynamics, pharmacokinetics, and safety of the oral reversible P2Y12 antagonist AZD6140 with aspirin in patients with atherosclerosis: a double-blind comparison to clopidogrel with aspirin. Eur Heart J. 2006;27:1038-47.

8. Cannon CP, Husted S, Harrington RA, et al. Safety, tolerability, and initial efficacy of AZD6140, the first reversible oral adenosine diphosphate receptor antagonist, compared with clopidogrel, in patients with non-ST-segment elevation acute coronary syndrome: primary results of the DISPERSE-2 trial. J Am Coll Cardiol. 2007;50:1844-51.

9. Wallentin L, Becker RC, Budaj A, et al. Ticagrelor versus clopidogrel in patients with acute coronary syndromes. N Engl J Med. 2009;361:1045-57.

10. Steg PG, James SK, Atar D, et al. ESC guidelines for the management of acute myocardial infarction in patients presenting with ST-segment elevation. The Task Force on the management of ST-segment elevation acute myocardial infarction of the European Society of Cardiology (ESC). Eur Heart J. 2012;33: 2569-619.

11. NewsRx. Medication Compliance: $40 \%$ of American adults report difficulty in swallowing pills. http://www.newsrx.com/ newsletters/Biotech-Business-Week/2004-02-09/0209200433345 2BB.html. Accessed 18 April 2013.

12. Carnaby-Mann G, Michael Crary M. Pill swallowing by adults with dysphagia. Arch Otolaryngol Head Neck Surg. 2005;131: 970-5.

13. Strachan I, Greener M. Medication-related swallowing difficulties may be more common than we realise. Pharm Pract. 2005; 15:411-4.

14. White R, Bradnam V. Handbook of drug administration via enteral feeding tubes. http://www.pharmacy.cmu.ac.th/unit/unit_files/files download/2012-03-26HandbkOfDrugAdminiViaEnteralFeeding Tubes\%201stEd_WhiteAndBradn.pdf Accessed 16 Jan 2013.

15. Chen ZM, Jiang LX, Chen YP, et al. Addition of clopidogrel to aspirin in 45,852 patients with acute myocardial infarction: randomised, placebo-controlled trial. Lancet. 2005;366:1607-21.

16. Storey RF, Husted S, Harrington RA, et al. Inhibition of platelet aggregation by AZD6140, a reversible oral P2Y12 receptor antagonist, compared with clopidogrel in patients with acute coronary syndromes. J Am Coll Cardiol. 2007;50:1852-6.

17. James S, Akerblom A, Cannon CP, et al. Comparison of ticagrelor, the first reversible oral $\mathrm{P} 2 \mathrm{Y} 12$ receptor antagonist, with clopidogrel in patients with acute coronary syndromes: rationale, design, and baseline characteristics of the PLATelet inhibition and patient Outcomes (PLATO) trial. Am Heart J. 2009;157: 599-605.

18. AstraZeneca. Data on file: clinical study D5130C00055.

19. AstraZeneca. Data on file: AstraZeneca formulation development report-taste prediction of ticagrelor by an electronic tongue. 